\title{
ZONIFICACIÓN DE LOS PROCESOS DENUDACIONALES EN LA MICRO-CUENCA DEL RÍO CRUZ, ESCAZÚ, SAN JOSÉ, COSTA RICA: RECOMENDACIONES PARA MEJORAR LA GESTIÓN DEL TERRITORIO
}

\author{
ZONING BASED ON DENUDATIONAL PROCESSES OF THE CRUZ RIVER \\ WATERSHED, ESCAZÚ, SAN JOSÉ, COSTA RICA: RECOMMENDATIONS TO \\ IMPROVE THE SPATIAL MANAGEMENT
}

\author{
Rolando Mora* \& Raquel Granados \\ Escuela Centroamericana de Geología, Universidad de Costa Rica \\ Apartado: 2142060UCR, San Pedro, San José, Costa Rica \\ *Autor para contacto: rmorach@gmail.com
}

(Recibido: 29/11//2013 ; aceptado: 25/03/2014)

\begin{abstract}
The zoning of the Cruz River watershed was performed using a combination of geological, geomorphological and topographic information, and the results of the calculation of the probability of landslide occurrence. With the results of this investigation a proposal of recommended land use is presented and suggestions are forwarded to the Local Government in relation to the granting of building permits in the various sectors.

Keywords: Natural hazards, prevention, zoning, landslides, risk management.

RESUMEN: La zonificación de la micro-cuenca del río Cruz se ha realizado mediante la combinación de información geológica, geomorfológica, topográfica y los resultados del cálculo de la probabilidad de ocurrencia de deslizamientos. Con los resultados obtenidos de esta investigación se presenta una propuesta de uso recomendado del terreno y se hacen llegar sugerencias al Gobierno Local en lo que respecta al otorgamiento de permisos de construcción en los diferentes sectores.

Palabras clave: Amenazas naturales, prevención, zonificación, deslizamientos, gestión del riesgo.
\end{abstract}




\section{UBICACIÓN}

Con el objetivo de realizar la zonificación de los procesos de la geodinámica externa, considerando aspectos geomorfológicos y la probabilidad de ocurrencia de deslizamientos, se ha abordado la microcuenca del río Cruz, la cual involucra la quebrada Lajas, sitio donde se ha presentado la mayor tragedia en la historia de Costa Rica, en lo que respecta a muertes causadas por un evento de deslizamiento.

La microcuenca del río Cruz se localiza en el cantón de Escazú, Provincia de San José, comprende un área de $6,35 \mathrm{~km}^{2}$, abarcando el río del mismo nombre, las quebradas Lajas e Higuerones, y algunas otras sin nombre (Fig. 1). Forman parte de la misma microcuenca los cerros Tapezco y Bandera, así como varias comunidades del cantón de Escazú.

\section{MARCO GEOMORFOLÓGICO}

El área comprendida por la microcuenca del río Cruz ha sido dividida en nueve unidades geomorfológicas (Fig. 2), de acuerdo con su génesis y siguiendo el método de clasificación de vanZuidam (1986), a continuación se describe cada una de las unidades:

D1 Montañas y colinas denudacionales: Topografía que comprende desde planicies $\left(0^{\circ}\right.$ $2^{\circ}$ ), como la cima del cerro Tapezco, pendientes de muy bajas hasta muy fuertes, en las divisorias de aguas, hasta pendientes extremadamente fuertes $\left(>55^{\circ}\right)$, hacia la cabecera y los márgenes de las quebradas, con procesos denudacionales especialmente severos en la parte alta de la quebrada Lajas. Esta unidad abarca la parte alta de la microcuenca, bordea las cabeceras de los ríos y quebradas y corresponde, principalmente, con las divisorias de las microcuencas que forman parte de la cuenca del río Cruz, también involucra algunos sectores donde se desarrollan eventos de caídas de rocas y deslizamientos, los cuales no son cartografiables a la escala de este trabajo.

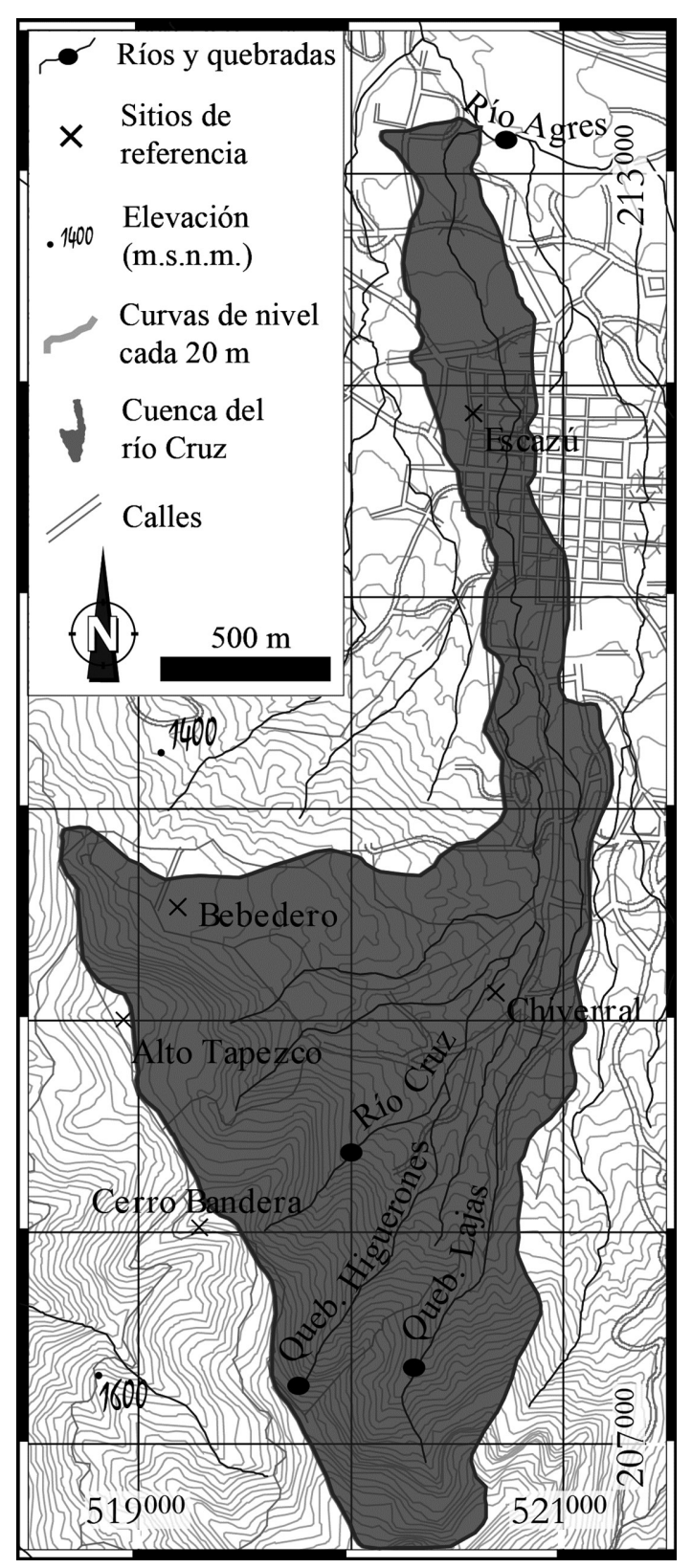

Fig. 1:Ubicación de la microcuenca del río Cruz, se muestran los nombres de los ríos, quebradas y puntos de interés que se encuentran presentes en las figuras posteriores.Parte de la hoja Abra 1:50 000, coordenadas Lambert Costa Rica Norte. 
D2Depósitos coluvio-aluvionales depiedemonte: Topografía que va desde planicies $\left(0^{\circ}-2^{\circ}\right)$, en las cercanías de la confluencia del río Cruz con el río Agres, hasta pendientes de muy bajas a moderadas en la mayor parte de la unidad, así como, pendientes muy fuertes $\left(35^{\circ}\right.$ $55^{\circ}$ ), sujetas a procesos de denudación intensos, en las áreas más cercanas a los cauces de ríos y quebradas. Esta unidad se ha desarrolladoa partir de ladepositacióncoluvial y aluvial de materiales provenientes de la parte alta de la cuenca y se extiende desde la base de los cerros de mayor altitud de la microcuenca hasta las partes baja de la misma. El patrón de drenaje en la unidad es de subdentrítico a subparalelo, con varias corrientes que corren paralelas entre sí,presentando un tributario mayor (río Cruz) bien definido y de caudal mayor, a donde confluye una serie de afluentes pequeños (queb. Higuerones, Lajas, y las quebradas sin nombre)(Fig. 1).

D3Áreas afectadas severamente por movimientos en masa: Topografía con pendientes desde bajas $\left(4^{\circ}-8^{\circ}\right)$, en el cauce de la quebrada Lajas, hasta pendientes extremadamente fuertes $\left(>55^{\circ}\right)$, la mayor parte de esta forma del terreno corresponde con pendientes de $16^{\circ}$ a $35^{\circ}$ ). Esta unidad comprende la parte alta de la microcuenca de la quebrada Lajas, en la que existe un gran número de deslizamientos no mapeables por su tamaño, los cuales se clasifican como deslizamientos traslacionales, así como, caída de rocas. La unidad involucra el contacto entre las Cornubianitas de Escazú y el Intrusivo de Escazú. Esta zona es propensa a desarrollar flujos de lodo y rocas, como el ocurrido el 3 de noviembre de 2010, producto del cual fallecieron 24 personas en un solo evento.

D4 Deslizamiento inactivo: Topografía con pendientes desde bajas $\left(4^{\circ}-8^{\circ}\right)$ hasta muy fuertes $\left(35^{\circ}-55^{\circ}\right)$, sujetas, en algún momento, a procesos de denudación intensos y deslizamientos. No se aprecian indicios de actividad reciente, sin embargo,

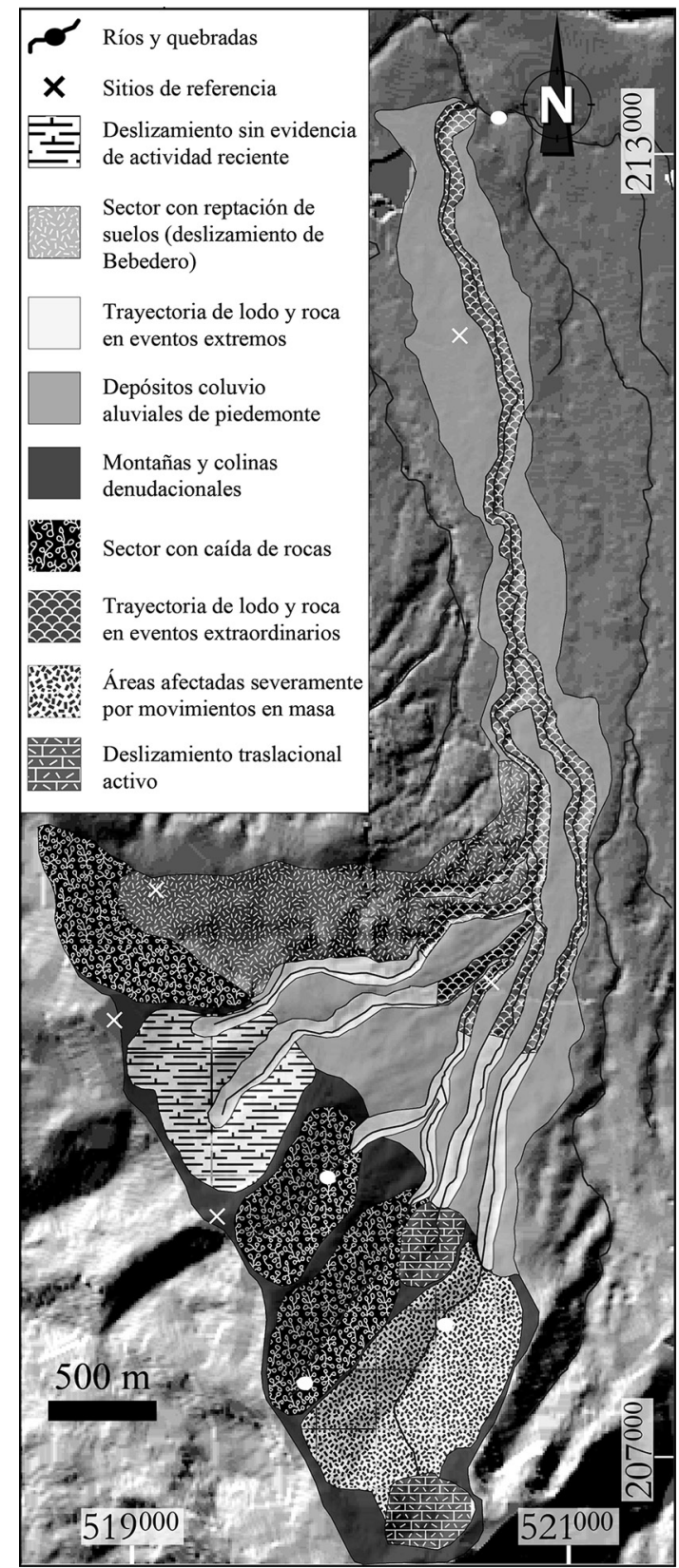

Fig. 2: Mapa Geomorfológico de la microcuenca del río Cruz. Parte de la hoja Abra 1: 50000 y de las hojas 1: 10000 Escazú, Pavas y Real de Pereira. Coordenadas Lambert Costa Rica Norte. 
no se descarta una posible reactivación del movimiento. Esta unidad corresponde con la parte alta de las microcuencas de dos quebradas sin nombre, que se ubican al SE de Bebedero.

D5 Sector con reptación de suelos (Deslizamiento Bebedero): Topografía que comprende desde planicies $\left(0^{\circ}-2^{\circ}\right)$, que corresponden con el cuerpo del deslizamiento Bebedero, hasta pendientes muy fuertes $\left(35^{\circ}-55^{\circ}\right)$, sujetas a procesos de denudación intensos en la parte cercana a la corona del mismo deslizamiento. En esta unidad se desarrolla un proceso de reptación poco profunda, la misma no se encuentra disectada, ya que no se desarrollan cauces en su superficie, sin embargo, las aguas de escorrentía superficial favorecen la erosión laminar. El espesor de la capa que repta es inferior a $15 \mathrm{~m}$, por lo que las edificaciones que están sobre esta forma del terreno se pueden ver afectadas.

D6Sector con caída de rocas: Topografía de pendientes desde bajas $\left(4^{\circ}-8^{\circ}\right)$, en el área cercana a la divisoria de aguas, hasta pendientes muy fuertes $\left(35^{\circ}-55^{\circ}\right)$, sujeta a procesos de denudación intensos al noreste del Alto Tapezco. Comprende la parte alta de las microcuencas del río Cruz y las quebradas Higuerones, donde se presenta la caída de rocas de la Formación Pacacua y del Intrusivo de Escazú.

Lechos menores sujetos a flujos de lodo y rocas: Topografía que va desde planicies $\left(0^{\circ}\right.$ $2^{\circ}$ ), al norte de la cuenca, hasta pendientes muy fuertes $\left(35^{\circ}-55^{\circ}\right)$, en las partes altas de la cuenca. Esta unidad corresponde con el lecho menor de los cauces de todos los ríos y quebradas que conforman la cuenca del río Cruz, así como el área de inundación de las mismas, por donde pueden discurrir flujos de lodo y rocas, debido a que en la parte alta se presentan procesos de remoción en masa y caída de rocas. Al sureste del Alto Tapezco se observan depresiones que bordean el cauce de las dos quebradas que nacen allí, en estos sitios se desarrollan desprendimientos de rocas y suelos, que al encausarse por la red fluvial, pueden generar flujos de lodo y rocas, los cuales son de alta energía debido a la altura del sitio donde se originan, la pendiente pronunciada del terreno y el reducido ancho de los cauces. Esta unidad se divide en D7 y D8, considerando el evento de precipitaciones extremas que generó un flujo de lodo y rocas el 3 de noviembre del 2010, por lo que la altitud a la que llegó el mismo marca la diferencia entre la posible trayectoria de flujos de lodo y rocas en eventos extremos (D7), de la posible trayectoria de flujos de lodo y rocas en eventos extraordinarios (D8). El evento ocurrido el 3 de noviembre del 2010 corresponde con una probabilidad baja de ocurrencia, por lo que un evento extraordinario tendría una probabilidad aún más baja de ocurrir.

D9 Deslizamiento traslacional activo: Existen dos deslizamientos, uno corresponde con la parte alta de la microcuenca de la quebrada Lajas, y el otro con la microcuenca de una quebrada que se encuentra entre las quebradas Lajas e Higuerones.

El primero presenta una topografía con pendientes desde moderadas $\left(8^{\circ}-16^{\circ}\right)$ hasta extremadamente fuertes $\left(>55^{\circ}\right)$, mientras que el segundo presenta una topografía con pendientes desde moderadas $\left(8^{\circ}-16^{\circ}\right)$ hasta muy fuertes $\left(35^{\circ}-55^{\circ}\right)$. Los deslizamientos involucran la capa superficial de suelo residual, la cual es de poco espesor, sin embargo, como se observó en el evento del 3 de noviembre del 2010, las consecuencias pueden ser muy importantes, debido a que los flujos desarrollados a partir de ese evento pueden ser altamente energéticos.

\section{MARCO GEOLÓGÍCO}

En la cuenca del río Cruz afloran varias formaciones geológicas, las cuales fueron observadas en el campo y se incluyen en el mapa (Fig. 3 ). A continuación se describen de la más antigua a la más reciente. 
Formación Pacacua: Según Hernández \& Vargas (1992) aflora en los cerros Tapezco, Granadilla, Alto Palomas, Alto Raicero, en la localidad de Bello Horizonte y en varios sectores de los cauces de los ríos Agres y Cruz; las rocas que la componen son areniscas compactas de color verde, sanas y de color morado alteradas, brechas deleznables constituidas por fragmentos de lavas y tobas, flotando en una matriz arcillosa, y conglomerados fosilíferos heterogéneos con fósiles poco preservados, clastos de lava grisácea y de tobas blancas.

La evaluación del espesor de los afloramientos, en los cerros Bandera y Tapezco y la información de pozos indican un espesor de $1000 \mathrm{~m}$ aproximadamente. El contacto inferior no aflora en Escazú. Su contacto superior es transicional hacia la Formación Peña Negra. Rivier (1979) le asigna una edad Mioceno Medio con base en microfauna.

En la cuenca del Río Cruz la Formación Pacacua aflora en la parte SW, en la parte alta de la misma, además, es posible observar afloramientos de esta formación cuando se producen deslizamientos y la cobertura de depósitos coluvio-aluviales ha sido removida. Estos sitios no aparecen en el mapa geológico debido a la pequeña escala de los mismos.

Formación Peña Negra: Su extensión es menor que la de la Formación Pacacua, presentándose en el Cerro Bandera, en la confluencia del río Uruca con la Quebrada Yeguas y en las cercanías al Alto Palo Campano. Está compuesta por una sucesión de unos $200 \mathrm{~m}$ de espesor de lutitas negras silíceas (lodolitas), compuestas por granos de minerales y microfósiles, flotando en una matriz arcillosa y areniscas medias de color café claro a amarillento, con una pátina café de meteorización, sin estructuras sedimentarias visibles, de textura microgranular, porosas y deleznables, muy diaclasadas, con algunas pátinas de manganeso cubriendo las diaclasas (Hernández \& Vargas, 1992).

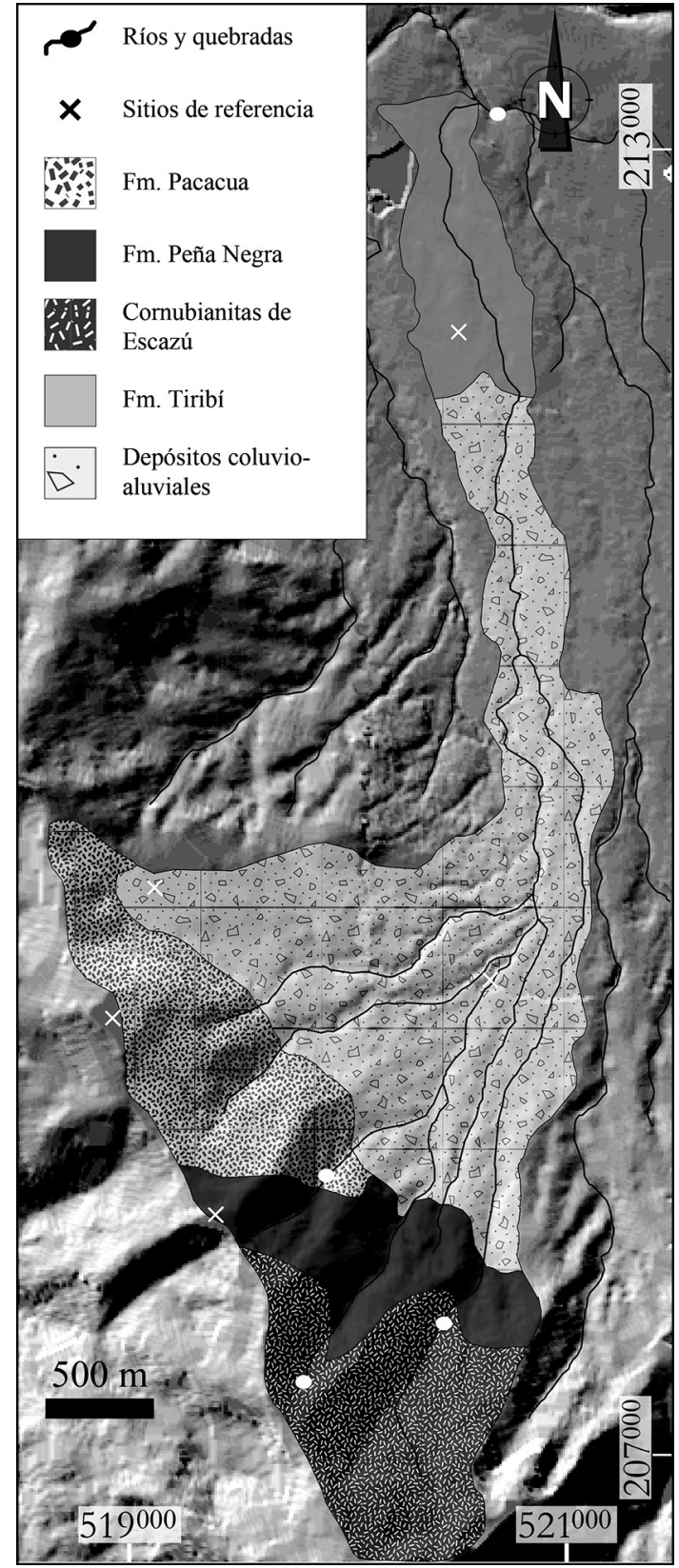

Fig.3: Mapa Geológico de la microcuenca del río Cruz. Parte de la hoja Abra 1: 50000 y de las hojas 1: 10000 Escazú, Pavas y Real de Pereira. Coordenadas Lambert Costa Rica Norte. 
El contacto inferior es transicional con la Formación Pacacua y el superior es difícil de identificar por la ausencia de buenos afloramientos (Hernández \& Vargas, 1992). Rivier (1979) le asigna una edad Mioceno Medio tardío Mioceno Medio Superior, lo cual fue confirmado por Hernández \& Vargas (1992) mediante análisis micropaleontológico.

En la cuenca del Río Cruz se encontró esta formación extendiéndose en sentido W-SE. En sitios cercanos a la quebrada Higuerones se encontraron algunas muestras de roca correlacionables con esta formación, que presentan aparente silicificación, lo cual podría estar relacionado con la influencia del intrusivo que dio origen a las cornubianitas.

Formación Monzonita Gabro de Escazú: Según Hernández \& Vargas (1992) esta formación aflora en el Cerro Cedral, Quebrada Londres y en la confluencia del Río Agres con esta quebrada, en la Quebrada Higuerones y en la Quebrada Lajas.

Se manifiesta como cuerpos discordantes con la Formación Pacacua y como cuerpos concordantes con la Formación Peña Negra, macroscópicamente se clasifican como gabros y monzonitas con textura holocristalina y granular, con plagioclasas, biotitas, y epidota como mineral de alteración (Hernández \& Vargas, 1992). Es posible encontrar rocas con textura porfirítica, semejantes a lavas (Hernández \& Vargas, 1992). Microscópicamente se clasifican como Monzogabros, Monzonitas y Dioritas (Hernández \& Vargas, 1992). Saenz (1982) le asigna una edad de 7,2 ma.

En las visitas realizadas al campo no fue posible encontrar afloramientos de esta formación, posiblemente porque se encuentra en profundidad y aflora únicamente en cauces de quebradas. Para efectos del tipo de análisis realizado en este estudio, no se tomó en cuenta esta formación, por lo que no aparece en el mapa geológico ni se utilizaron los datos geotécnicos correspondientes con la misma.

Cornubianitas de Escazú: Esta formación se encuentra bien expuesta en el Cerro Pico Blanco, en las partes altas de la Quebrada Higuerones y la
Quebrada Lajas, así como, en el cauce superior del Río Agres. Son rocas duras, muy resistentes a la meteorización, con fractura concoidea, que preservan estructuras primarias (estratificación, trazas fósiles), con minerales de alteración como sericita, calcita, muscovita y ceolitas (Hernández \& Vargas, 1992). Denyer \& Arias (1991) agrupan dentro de esta formación a todas las rocas del Valle Central afectadas por el metamorfismo causado por el emplazamiento del Intrusivo de Escazú en el Mioceno Superior-Plioceno, asignándolesla misma edad.

Formación Tiribí: Depósitos de ignimbritas, compuestos por pómez con fragmentos líticos y una matriz de ceniza(Pérez, 2000). Pérez et al (2006) le asignan una edad de 322000 años.

Coluvios y aluviones: Según Hernández \& Vargas (1992 estos depósitos son producto de la erosión y meteorización de las rocas preexistentes, su espesor es de $25 \mathrm{~m}$ aproximadamente, sobreyacen discordantemente a la Formación Pacacua. Los coluvios están constituidos por bloques angulares, mientras que los aluviones por clastos redondeados, ambos presentan clastos de intrusivo, cornubianitas y rocas sedimentarias de tamaño variable y englobados en una matriz arenosa (Hernández \& Vargas, 1992). Denyer\& Arias (1991) mencionan los abanicos coluvio-aluviales que sobresalen bordeando los cerros de Escazú.

\section{ANÁLISIS DE ESTABILIDAD DE LADERAS Y CÁLCULO DE LA PROBABILIDAD DE OCURRENCIADE DESLIZAMIENTOS}

Para analizar la estabilidad de las laderas en condiciones estáticas y calcular la probabilidad de ocurrencia de deslizamientos en condiciones pseudo-estáticas, se ha utilizado el programa de cómputo PISA (Probabilistic Infinite Slope Analysis) (Haneberg, 2005), el cual ejecuta cálculos probabilísticos de estabilidad de taludes, en condiciones estáticas y pseudo-estáticas, a partir de modelos de elevación digital del terreno, utilizando la ecuación del talud infinito y funciones de probabilidades para cada uno 
delos parámetros que la integran.

Parámetros de entrada: El modelo de elevación digital se ha generado a partir de la digitalización de las curvas de nivel de las Hojas Topográficas Escazú, Pavas y Real de Pereira escala 1:10 000, así como las de la Hoja Topográfica Abra escala 1:50 000, esto últimopara cubrir las áreas que no se encuentran a escala 1:10 000. Las elevaciones topográficas son representadas con intervalos de 20,0 m y el tamaño de la rejilla utilizada para la confección del modelo, y para la realización de los mapas y cálculos posteriores, es de $10 \mathrm{~m}$ x $10 \mathrm{~m}$.

Los parámetros de resistencia al corte de los suelos presentes en el área de estudio se obtuvieron mediante la realización de ensayos de corte directo en muestras inalteradas, así como a partir de la recopilación de información bibliográfica.

El ángulo de fricción interna de los suelos pertenecientes a la Formación Pacacua presenta una distribución de tipo triangular y oscila entre $21^{\circ}$ y $28^{\circ}$, con un pico de $25^{\circ}$. La cohesión de los suelos de esta formación también se considera como una distribución triangular, y sus valores oscilan entre $19000 \mathrm{~Pa}$ y $25000 \mathrm{~Pa}$, con un pico de $22000 \mathrm{~Pa}$. Según las observaciones realizadas en las visitas de campo y los datos recopilados de trabajos anteriores, se ha establecido que el espesor de material deslizable puede oscilar entre 20 m y $80 \mathrm{~m}$, y se le ha asignado una distribución uniforme. Con lo anterior se pretende considerar únicamente deslizamientos superficiales, similares al ocurrido el 3 de noviembre de 2010.

La Formación Peña Negra presenta una distribución uniforme de los parámetros de resistencia, con el ángulo de fricción interna oscilando entre $33^{\circ}$ y $35^{\circ}$, y una cohesión de $5000 \mathrm{~Pa}$. Según las observaciones realizadas en las visitas de campo y los datos recopilados de trabajos anteriores, se ha establecido que el espesor deslizable puede oscilar entre $1 \mathrm{~m}$ y $8 \mathrm{~m}$, y se le ha asignado una distribución uniforme.

Se obtuvo un ángulo de fricción interna de $40^{\circ}$ y una cohesión de 8500 Pa para la formación Cornubianitas Escazú, a ambos parámetros se les asignó una distribución uniforme. Según las observaciones realizadas en las visitas de campo y los datos recopilados de trabajos anteriores, se ha establecido que el espesor deslizable puede oscilar entre $1 \mathrm{~m}$ y $8 \mathrm{~m}$, y se le ha asignado una distribución uniforme.

Los suelos derivados de la formación Avalancha Ardiente, presentan distribuciones uniformes para el ángulo de fricción interna $\left(31^{\circ}\right.$ $\left.36^{\circ}\right)$ y la cohesión (1 $\left.000 \mathrm{~Pa}-36000 \mathrm{~Pa}\right)$. Según las observaciones realizadas en las visitas de campo y los datos recopilados de trabajos anteriores, se ha establecido que el espesor deslizable puede oscilar entre $1 \mathrm{~m}$ y $3 \mathrm{~m}$, y se le ha asignado una distribución uniforme.

Los Depósitos Aluvionales y Coluviales presentan una distribución de tipo triangular, tanto en su ángulo de fricción interna como en la cohesión, oscilando entre $26^{\circ}$ y $36^{\circ}$, con un pico de $31^{\circ}$, y entre $0 \mathrm{~Pa}$ y $25000 \mathrm{~Pa}$, con un pico de $12500 \mathrm{~Pa}$, respectivamente. Según las observaciones realizadas en las visitas de campo y los datos recopilados de trabajos anteriores, se ha establecido que el espesor deslizable puede oscilar entre $1 \mathrm{~m}$ y 8 $\mathrm{m}$, y se le ha asignado una distribución uniforme.

Debido a que no se da la presencia de niveles de agua en los suelos, el coeficiente de presión de poros no se ha tomado en consideración, pero el efecto de pérdida de resistencia por humedecimiento se ha incluido al realizar los ensayos de corte directo en condiciones de saturación total.

Tanto el peso unitario seco como el húmedo y el saturado presentan distribuciones de tipo uniforme, por lo que sus valores se resumen en el cuadro 1.

A partir de la combinación de los parámetros geotécnicos, el modelo de elevación digital del terreno, el mapa geológico y el mapa geomorfológico, se obtuvieron tres mapas: Media del Factor de Seguridad (MFS), Probabilidad de Ocurrencia de Deslizamientos (POD) y Uso Recomendado del Suelo (URS).

Cabe destacar que el mapa URS toma en cuenta la probabilidad de ocurrencia de deslizamientos, la geomorfología y topografía de la cuenca y los efectos del evento registrado el 3 de noviembre del 2010.

Mapa de la Media del Factor de Seguridad (MFS): Este mapa representa la 
Cuadro 1

Peso unitario según formación geológica.

\begin{tabular}{cccc}
\hline Formación & $\gamma_{\mathrm{s}}\left[\mathrm{kN} / \mathrm{m}^{3}\right]$ & $\gamma_{\mathrm{h}}\left[\mathrm{kN} / \mathrm{m}^{3}\right]$ & $\gamma_{\text {sat }}\left[\mathrm{kN} / \mathrm{m}^{3}\right]$ \\
\hline Pacacua & 10,6 & 15,6 & 16,6 \\
Peña Negra & 13,2 & 17,2 & 18,1 \\
Cornubianitas de Escazú & 24,1 & 25,5 & 25,6 \\
Tiribí & 11,9 & 15,4 & 18,9 \\
Depósitos Aluvionales y Coluviales & 14,2 & 18,1 & 18,7 \\
\hline
\end{tabular}

$\gamma_{\mathrm{s}}$ : peso unitario seco

$\gamma_{\mathrm{h}}$ : peso unitario húmedo

$\gamma_{\text {sat }}:$ peso unitario saturado

media del factor de seguridad (MFS) (Fig. 5), el mismo se ha calculado aplicando el código PISA en toda la cuenca del río Cruz. El cálculo incluye los datos geotécnicos, geológicos y la topografía de la cuenca. El mapa MFS se ha reclasificado de la siguiente manera:

- $\quad$ Estable: si el Factor de Seguridad es $\geq 1,4$,

- Moderadamente estable si el Factor de Seguridad se encuentra entre 1,2 y 1,4 ,

- Inestable si el Factor de Seguridad se encuentra entre 1-1,2 y

- $\quad$ Muy inestable si el Factor de Seguridad es $\leq 1$.

A continuación se presenta la figura 4 , que muestra el porcentaje del área total de la cuenca que corresponde con cada clase del mapa:

- $\quad$ el 58,3\% forma parte de la clase estable,

- un $2,4 \%$ es moderadamente estable,

- $\quad$ un $2,5 \%$ es inestable y

- $\quad$ un $36,8 \%$ muy inestable.

Mapa de Probabilidad de Ocurrencia de Deslizamientos (POD): El mapa POD representa la probabilidad de que el factor de seguridad sea menor a 1 (FS $\leq 1)$ para toda la cuenca (Fig. 7).La Probabilidad de Ocurrencia de Deslizamientos fue reclasificada como sigue:

- $\quad$ muy baja cuando es $\leq 20 \%$,

- baja si se encuentra entre $20 \%$ y $40 \%$,

- moderada entre $40 \%$ y $60 \%$,

- $\quad$ alta si existe una probabilidad entre $60 \%$ y $80 \%$ y
- muy alta si está entre el $80 \%$ y el $100 \%$.

La figura 6 muestra el porcentaje del área de la cuenca para cada clase:

- $\quad 52 \%$ del área corresponde con una probabilidad muy baja,

- $\quad 8 \%$ con baja,

- $\quad 5 \%$ con moderada,

- $5 \%$ es alta y

- $\quad 30 \%$ muy alta.

Mapa de uso recomendado del suelo (URS): Este mapa es el resultado de la integración de los mapas Geomorfológico, Geológico, POD y el Modelo de Elevación Digital del Terreno (Fig. 9). Para el mapa de URS se establecieron tres categorías: Desarrollo, Desarrollo Controlado y Conservación, las cuales se definen en el cuadro 2.

La categoría Desarrollo representa el 17\% del área de la cuenca, Desarrollo Controlado un $21 \%$ y Conservación un $62 \%$. La figura 8 muestra la el porcentaje del área total de la cuenca que corresponde con cada clase.

\section{OBSERVACIONES PARA LA UTILIZACIÓN CORRECTA DE LOS MAPAS}

En el Cantón de Escazú los daños materiales y las pérdidas de vidas ocasionadas por los eventos de inundaciones y/o deslizamientos no son infrecuentes, además, los costos para limpiar 


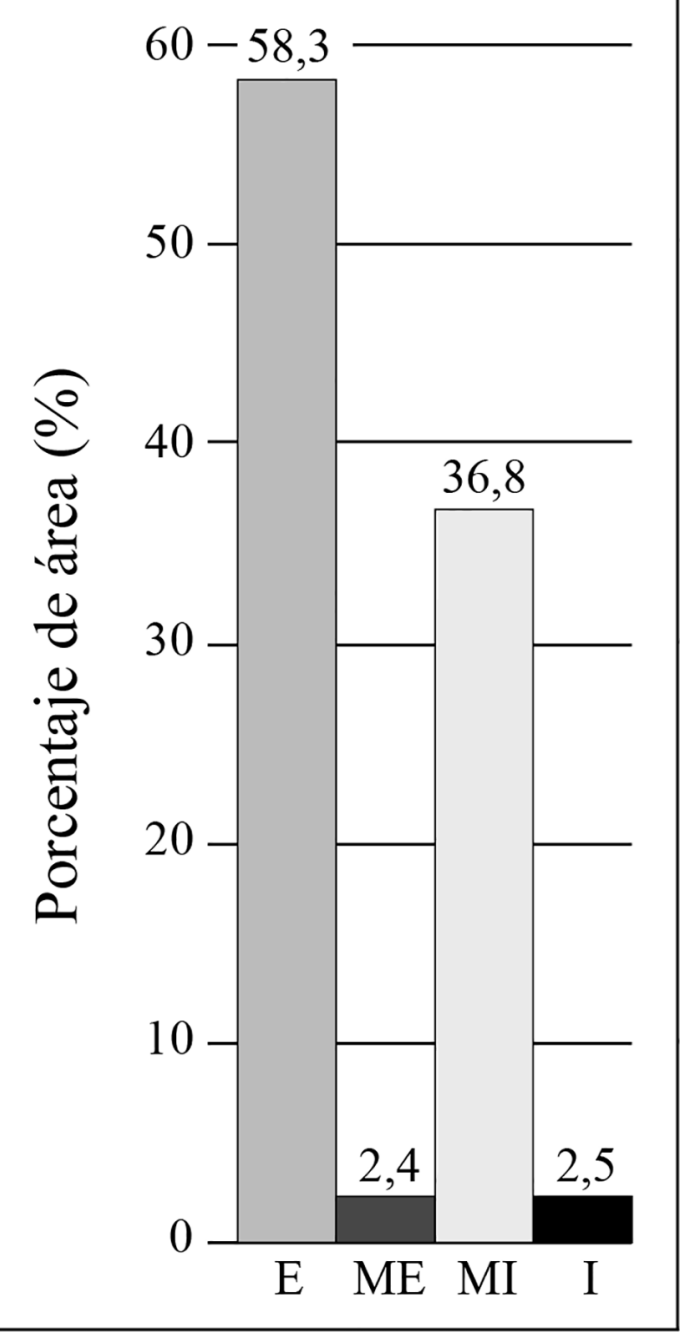

Fig. 4: Porcentaje del área de la cuenca del río Cruz que comprende cada clase de la media del factor de seguridad.

y reparar los impactos causados por estos eventos pueden ser considerables. Por lo anterior, la reducción de los daños a la propiedad privada y de la perturbación de los residentes por causa de los

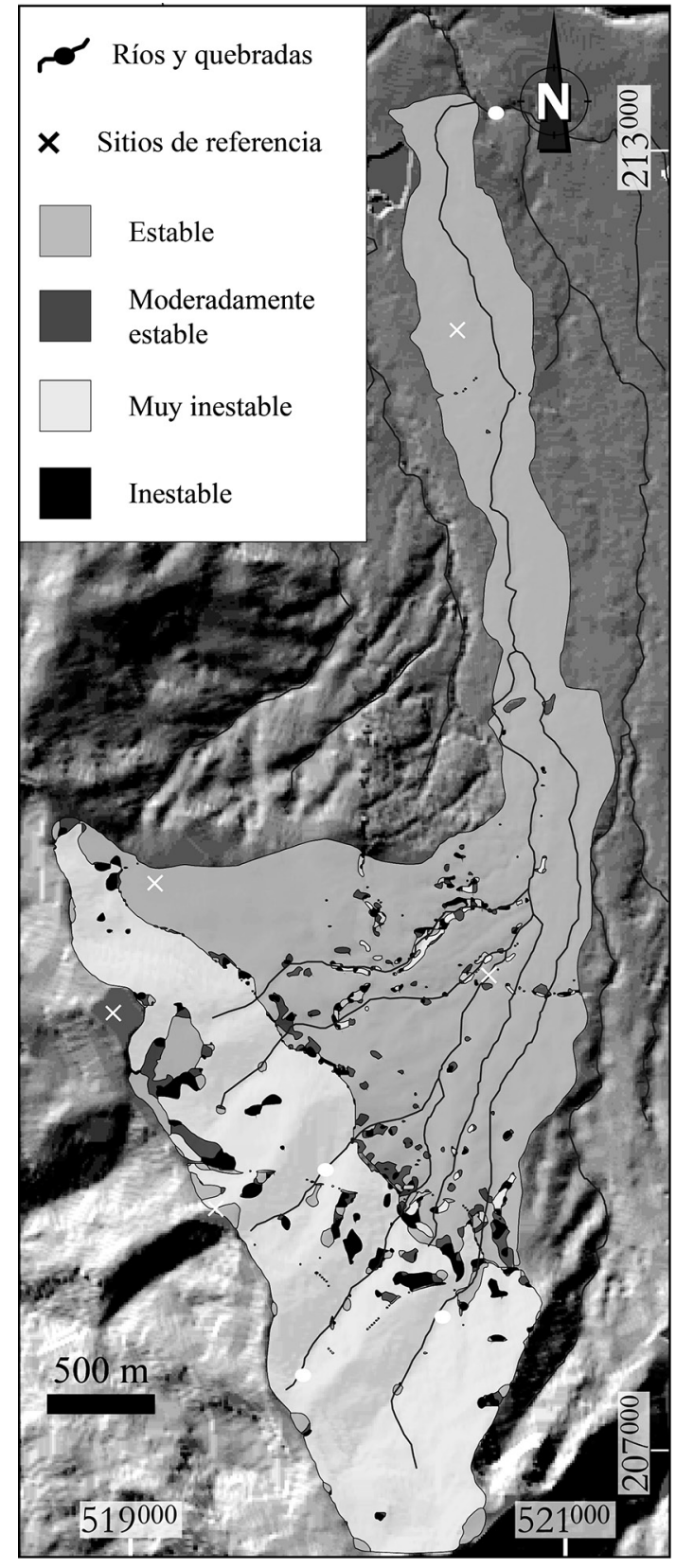

Fig.5: Mapa de la Media del Factor de Seguridad (MFS) de la cuenca del río Cruz. Parte de la hoja Abra 1: 50000 y de las hojas 1: 10000 Escazú, Pavas y Real de Pereira. Coordenadas Lambert Costa Rica Norte. 


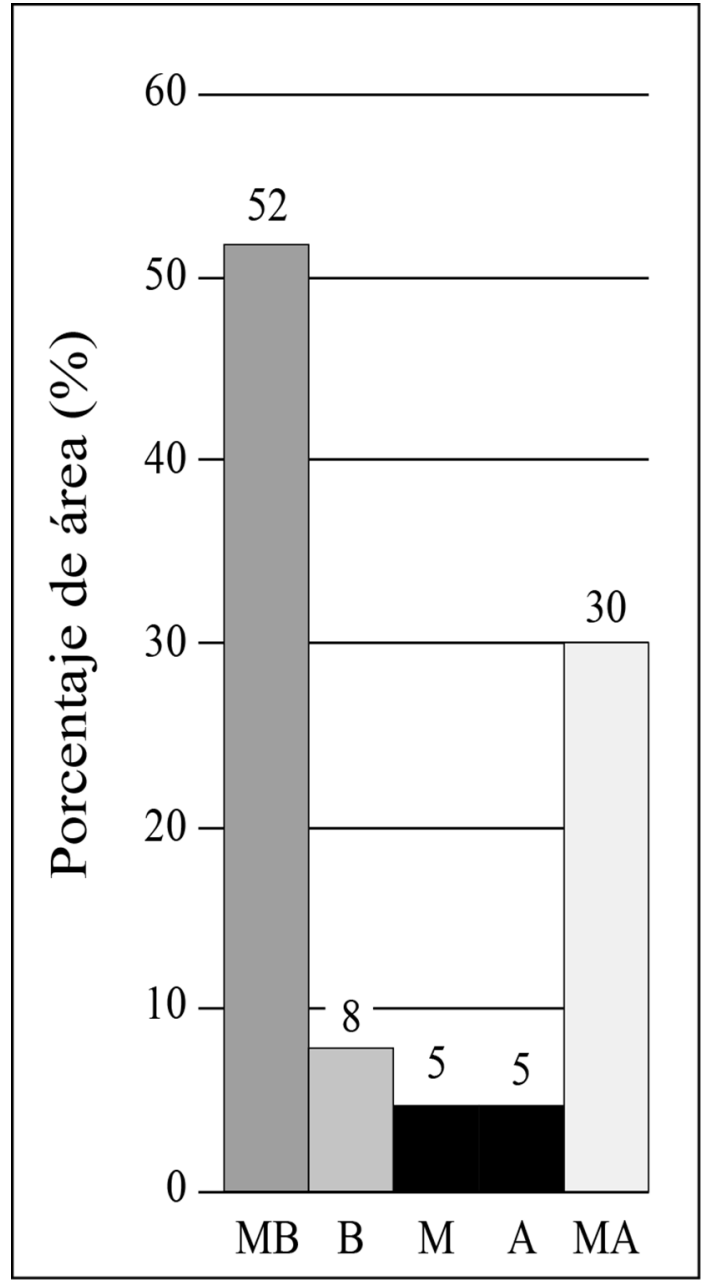

Fig. 6: Porcentajes de área cubiertos por cada clase de probabilidad de ocurrencia de deslizamientos de la cuenca del río Cruz.

eventos naturales debe ser una preocupación para el Gobierno Local, por lo cual, se le presenta la necesidad de asegurar que todos los nuevos desarrollos se realicen en sitios que estén a salvo del impacto de los eventos naturales o de una manera tal como para garantizar su seguridad ante estas amenazas.

La Municipalidad de Escazú debe exigir que los nuevos desarrollos, dentro del área cubierta por este estudio, y en general dentro de su juris-

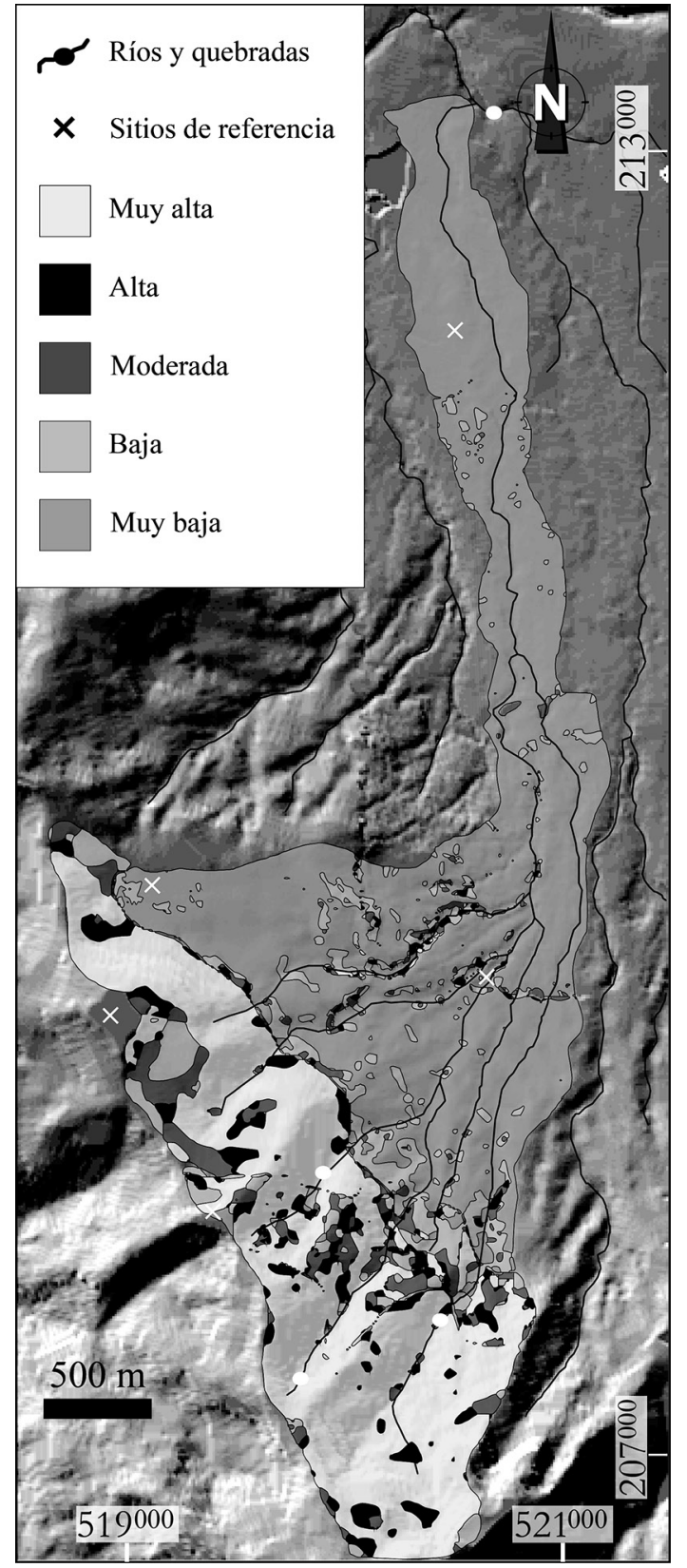

Fig.7: Mapa de Probabilidad de Ocurrencia de Deslizamientos en la cuenca del río Cruz. Parte de la hoja Abra 1: 50000 y de las hojas 1: 10000 Escazú, Pavas y Real de Pereira. Coordenadas Lambert Costa Rica Norte. 
Cuadro 2

Definición de las categorías de uso recomendado del suelo

\begin{tabular}{|c|c|}
\hline Categoría de uso & Características \\
\hline Desarrollo & $\begin{array}{l}\text { Sectores considerados aptos para el } \\
\text { desarrollo de infraestructura hab- } \\
\text { itacional e instalaciones esenciales. }\end{array}$ \\
\hline $\begin{array}{l}\text { Desarrollo } \\
\text { controlado }\end{array}$ & $\begin{array}{c}\text { Sectores donde se requieren } \\
\text { medidas correctivas para autorizar } \\
\text { su uso para el desarrollo de in- } \\
\text { fraestructura habitacional o de } \\
\text { instalaciones esenciales }\end{array}$ \\
\hline Conservación & $\begin{array}{l}\text { Sectores donde no se recomienda } \\
\text { la utilización del terreno para otro } \\
\text { fin que no sea el de la conservación } \\
\text { y la recuperación }\end{array}$ \\
\hline
\end{tabular}

dicción, tengan que ser planificados y construidos de tal manera que se minimice el impacto de los eventos naturales. En concreto, el Gobierno Local debe requerir que todo nuevo desarrollo debe estar ubicado en un terreno que es seguro para el uso previsto. Desde un punto de vista normativo, esto puede implicar:

- La delimitación los sectores bajo amenazas geológicas de todo el cantón, siguiendo criterios como los expuestos en esta investigación.

- Primordialmente, los sectores sujetos al impacto de los eventos naturales deben destinarse a la conservación del medio ambiente, el uso recreativo u otros usos no sensibles ante las amenazas.

- Solicitar estudios técnicos cuando se propongan desarrollos en los sectores clasificados como Desarrollo Controlado y Conservación.

- Exigir que se cumplan las condiciones para garantizar la seguridad de las personas y las obras, y realizar inspecciones a cargo de personal calificado, durante la construcción de los nuevos desarrollos en áreas potencialmente peligrosas.

Se debe recordar a los propietarios que en el momento de construir, el costo de la protección de una obra nueva es generalmente muy asequible, sin duda, es mucho menor que el costo de

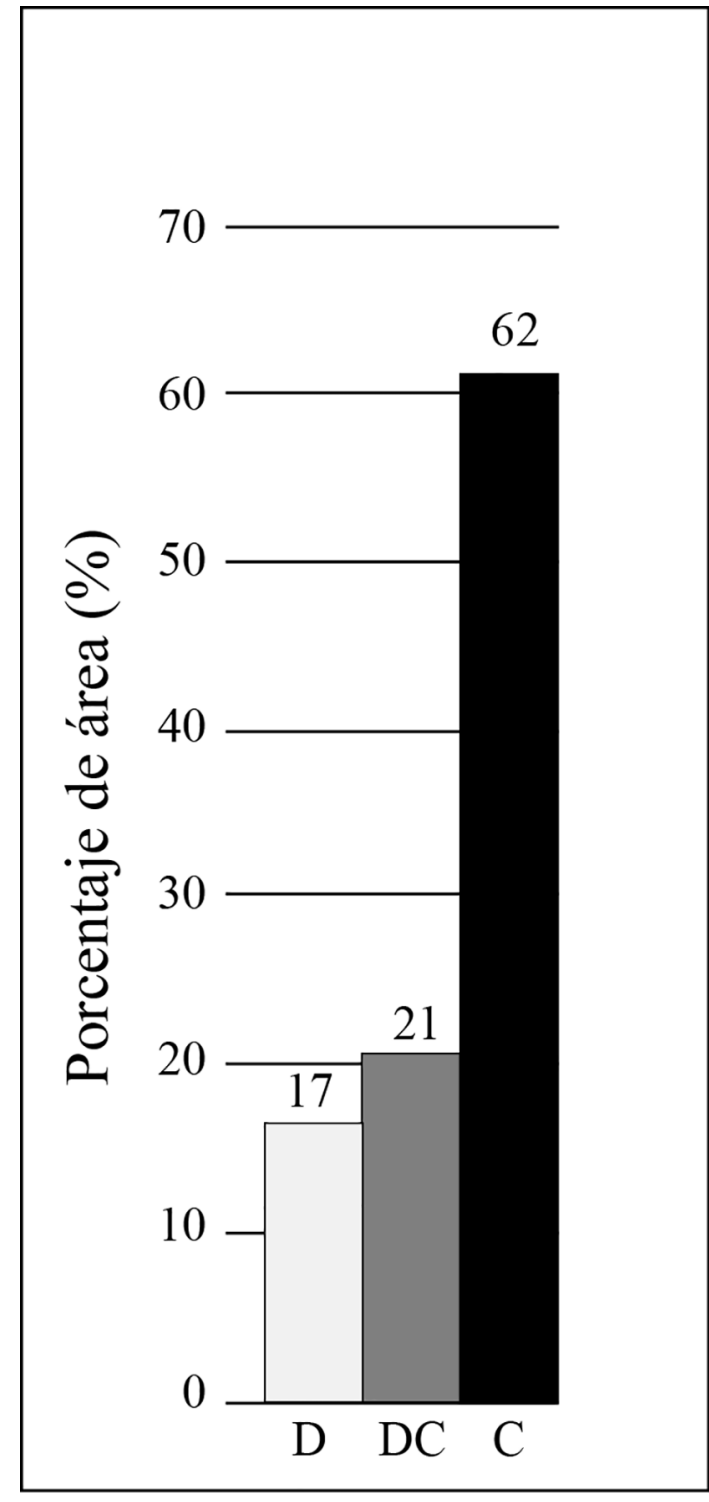

Fig.8: Porcentajes de área cubiertos por cada clase de probabilidad de ocurrencia de deslizamientos de la cuenca del río Cruz.

modificar o reubicar una obra ya existente, ya que las medidas de protección pueden ser implementadas en el inicio de la construcción.

Terrenos u obras que son anteriores a este proceso de aprobación, puede requerir un traba- 
jo de protección complejo y deben abordarse con cautela. En estos casos, puede ser necesario consultar con un profesional para determinar la forma más adecuada y económica para la protección de la propiedad.

Cuando un terreno propuesto para desarrollo se identifica como potencialmente sujeto a una amenaza geológica, el municipio debe pedir al propietario que presente un informe técnico elaborado por un profesional calificado. El informe, requerido por ordenanza municipal, debe demostrar que el terreno se puede utilizar de forma segura para el uso previsto. Como parte de este proceso de adquisición del permiso de construcción, se logran proteger los intereses del dueño de la propiedad, así como los de la comunidad en general y se asegura la construcción de estructuras seguras y sólidas.

La ordenanza municipal debe requerir que el propietario, que decida realizar la construcción de nuevos edificios o estructuras, las modificaciones estructurales de un edificio o estructura existente o la adición a un edificio o estructura existente, en un sitio que está sujeto o probablemente sujeto al impacto de amenazas geológicas, tiene que proporcionar un informe certificado por un profesional con experiencia en Geología, que certifique que el terreno puede usarse de manera segura para el uso previsto. En la mayoría de los casos, el informe puede recomendar medidas correctivas, que se deben cumplir en el transcurso del proyecto.

Es importante señalar, sin embargo, que si un informe técnico determina que el terreno no puede ser utilizado con seguridad para el uso previsto, el municipio debe negarse a expedir el permiso de construcción.

\section{CONCLUSIONES Y RECOMENDACIONES}

De acuerdo con los resultados obtenidos, el factor de seguridad predominante el área de estudio es el correspondiente con terrenos esta-

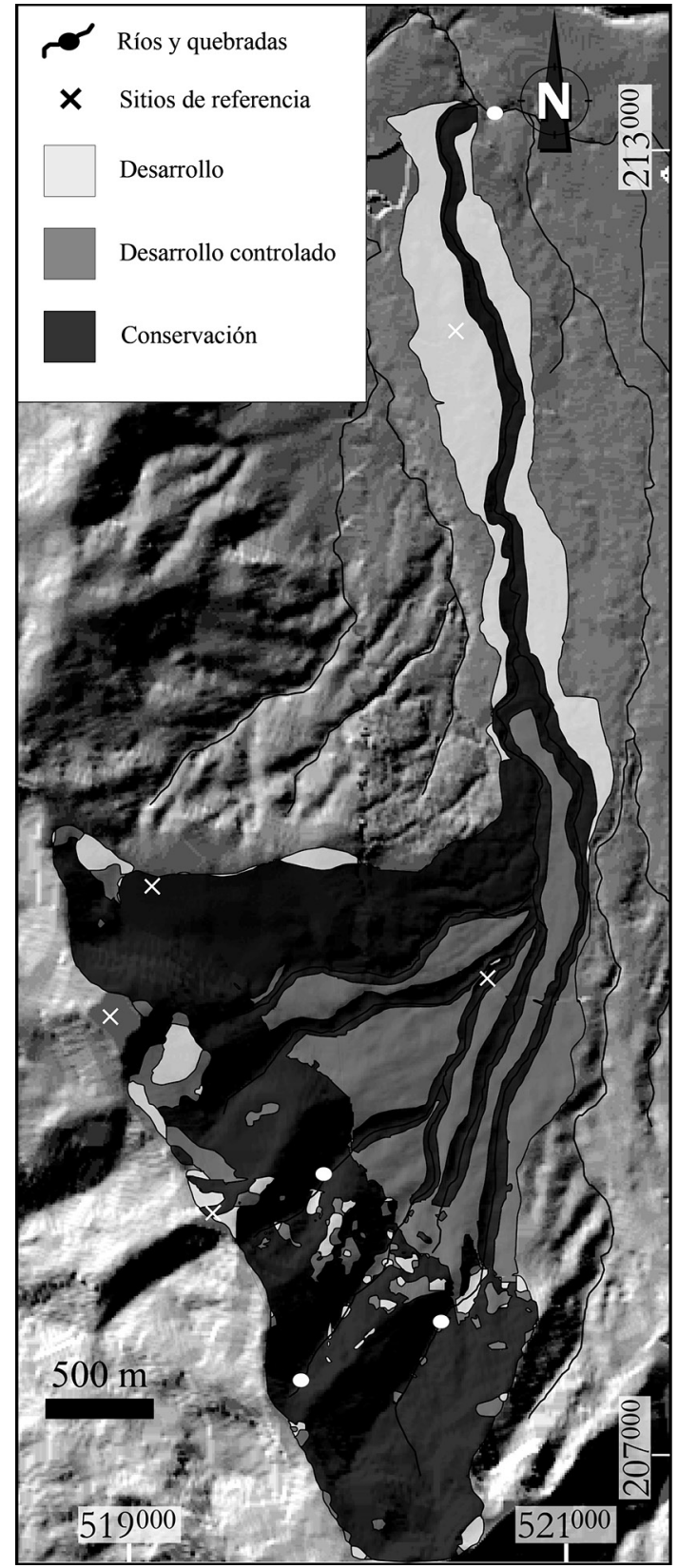

Fig.9: Mapa de Uso Recomendado del Suelo para la cuenca del río Cruz. Parte de la hoja Abra 1: 50000 y de las hojas 1: 10000 Escazú, Pavas y Real de Pereira. Coordenadas Lambert Costa Rica Norte. 
bles, pues el 58\% del área se encuentra dentro esta clasificación, sin embargo, es importante notar que más de un tercio del área $(36,8 \%)$ se presenta como muy inestable.

En lo que respecta a la probabilidad de ocurrencia de deslizamientos, un $60 \%$ del área se clasifica como de probabilidad de muy baja a baja, mientras que un $35 \%$ del área es de probabilidad de alta a muy alta.

El mapa de Uso Recomendado del Suelo es el resultado de la combinación de la información obtenida a partir de los mapas: Geológico, Geomorfológico, Media del Factor de Seguridad y Probabilidad de ocurrencia de Deslizamientos.

Considerando el mapa de Uso Recomendado del Suelo, el área estudiada debe destinarse a Desarrollo en un 17\%, Desarrollo Controlado en un $21 \%$ y Conservación en un $62 \%$. Es clara, entonces, la vocación de conservación predominante de la subcuenca del río Cruz.

De acuerdo con esta clasificación, los terrenos ubicados en las áreas designadas para el Desarrollo podrán ser utilizados siempre y cuando cumplan con los estudios rutinarios para la obtención de permisos de construcción, las áreas correspondientes con Desarrollo Controlado deberán contar con, además de los estudios rutinarios, evaluación de amenazas por profesionales calificados, de manera que se demuestre que el terreno el seguro para la construcción y/o se tomen las medidas necesarias para asegurar su estabilidad.

Las áreas que han sido designadas para la Conservación, corresponden con la integración de zonas con probabilidad de ocurrencia de deslizamientos alta a muy alta, zonas con reptación y/o deslizamientos, y zonas con posibilidad de generar o que permitan el descenso de flujos de lodo y rocas. Por esta razón, no se recomienda la utilización de estas áreas para el desarrollo de actividades humanas, sin embargo, los propietarios de los terrenos pueden presentar estudios técnicos para ser valorados por las autoridades municipales.
Es importante tener presente que el nivel de detalle de este trabajo es dependiente de la cartografía disponible a la fecha de su realización, siendo la escala 1:10 000 la más detallada. La toma de decisiones con base en la información generada en este estudio debe ser consecuente con la escala a la que va a ser aplicada.

Por lo tanto, en cuanto a la gestión territorial de la micro-cuenca del río Cruz, se recomienda el desarrollo de una base cartográfica más detallada, mediante la utilización de imágenes de alta resolución (por ejemplo LiDAR), e involucrando a la $\mathrm{CNE}$ y otros actores interesados en realizar esfuerzos conjuntos para la generación de la información requerida.

\section{REFERNCIAS BIBLIOGRÁFICAS}

DENYER, P. \& ARIAS, O., 1991: Estratigrafía de la Región Central de Costa Rica.- Rev. Geol. de Amér. Central, 12:1-59.

HANEBERG, W.C, 2005: PISA: Probabilistic infinite slope analysis, version 1.0 user manual.- 15 págs. Haneberg Geoscience.

HERNÁNDEZ, J. \& VARGAS, A., 1992: Geología urbana del cantón de Escazú, provincia de San José, Costa Rica.- 172 págs. Univ. de Costa Rica, San José [Campaña Bach.].

PÉREZ, W., ALVARADO, G.E.\& GANS, P.B., 2006: The 322 kaTiribí Tuff: stratigraphy, geochrology and mechanisms of deposition of the largest and most recent ignimbrite in the Valle Central, Costa Rica.- Bull. Volcanol. 69: 25-40.

PÉREZ, W., 2000: Vulcanología y petroquímica del evento ignimbrítico del Pleistoceno medio $(0,33 \mathrm{Ma})$ del valle central de Costa Rica.- 170 págs. Univ. de Costa Rica, San José [Tesis Lic.]. 
RIVIER, F., 1979: Geología del Área norte de los Cerros de Escazú, Cordillera de Talamanca - Costa Rica.- 38 págs. [Inf. interno].
VAN ZUIDAM, R.A., 1986: Aerial photo-interpretation in terrain analysis and geomorphologic mapping.- 442 págs. Smits Publishers, The Hague. 